\title{
Super Accelerated Motion in Rindler Spacetime
}

\section{Sangwha-Yi}

Department of Math, Taejon University, South Korea

\section{*Corresponding Author: Sangwha-Yi, Department of Math, Taejon University, South Korea}

Abstract: In the general relativity theory, we discover formulas that the super accelerated matter moves with the acceleration $\hat{\vec{b}}_{0}$ about Rindler space-time. We can represent the super accelerated motion about coordinates $X, c t, \xi^{0}$. PACS Number:04,04.90.+e

Keywords: General relativity theory, Super accelerated motion, Rindler spacetime

\section{INTRODUCTION}

In the general relativity theory, we discover formulas that the super accelerated matter moves with the acceleration $a_{0}$ about Rindler space-time.

At first, Rindler coordinate is

$$
\begin{aligned}
& c t=\left(\frac{c^{2}}{a_{0}}+\xi^{1}\right) \sinh \left(\frac{a_{0} \xi^{0}}{c}\right) \\
& x=\left(\frac{c^{2}}{a_{0}}+\xi^{1}\right) \cosh \left(\frac{a_{0} \xi^{0}}{c}\right)-\frac{c^{2}}{a_{0}} \\
& y=\xi^{2}, z=\xi^{3}
\end{aligned}
$$

In Eq (1),

$$
\xi^{1}+\frac{c^{2}}{a_{0}}=\frac{c t}{\sinh \left(\frac{a_{0} \xi^{0}}{c}\right)}
$$

If we insert $\mathrm{Eq}(4)$ in $\mathrm{Eq}(2)$,

$$
x=\operatorname{ctcoth}\left(\frac{a_{0} \xi^{0}}{c}\right)-\frac{c^{2}}{a_{0}}
$$

If we insert $\mathrm{Eq}(5)$ in $\mathrm{Eq}(2)$,

$$
\operatorname{ctcoth}\left(\frac{a_{0} \xi^{0}}{c}\right)=\left(\frac{c^{2}}{a_{0}}+\xi^{1}\right) \cosh \left(\frac{a_{0} \xi^{0}}{c}\right)
$$

Hence, the result is

$$
\xi^{1}=\frac{c t}{\sinh \left(\frac{a_{0} \xi^{0}}{c}\right)}-\frac{c^{2}}{a_{0}}
$$




\section{The Super ACCELERATED Motion AbOUT An Uniformly ACCELERATED Frame}

$d \tau^{2}=\left(1+\frac{a_{0} \xi^{1}}{c^{2}}\right)^{2}\left(d \xi^{0}\right)^{2}-\frac{1}{c^{2}}\left[\left(d \xi^{1}\right)^{2}+\left(d \xi^{2}\right)^{2}+\left(d \xi^{3}\right)^{2}\right]$

Hence, if the super accelerated matter moves with the acceleration $a_{0}{ }^{\prime}$ about an uniformly accelerated frame,

$$
a_{0}{ }^{\prime}=\frac{d}{d \xi^{0}}\left[\frac{\left(\frac{d \xi^{1}}{d \xi^{0}}\right)}{\sqrt{\left(1+\frac{a_{0} \xi^{1}}{c^{2}}\right)^{2}-\left(\frac{d \xi^{1}}{d \xi^{0}}\right)^{2} / c^{2}}}\right]
$$

If we compute,

$$
\left(a_{0}^{\prime} \xi^{0}\right)^{2}=\frac{\left(\frac{d \xi^{1}}{d \xi^{0}}\right)^{2}}{\left(1+\frac{a_{0} \xi^{1}}{c^{2}}\right)^{2}-\left(\frac{d \xi^{1}}{d \xi^{0}}\right)^{2} / c^{2}}
$$

Then,

$$
\left(\frac{d \xi^{1}}{d \xi^{0}}\right)^{2}=\left(a_{0}^{\prime} \xi^{0}\right)^{2}\left[\left(1+\frac{a_{0} \xi^{1}}{c^{2}}\right)^{2}-\left(\frac{d \xi^{1}}{d \xi^{0}}\right)^{2} / c^{2}\right]
$$

If we compute about $\frac{d \xi^{1}}{d \xi^{0}}$,

$$
\frac{d \xi^{1}}{d \xi^{0}}=\frac{a_{0}^{\prime} \xi^{0}\left(1+\frac{a_{0} \xi^{1}}{c^{2}}\right)}{\sqrt{1+\frac{\left(a_{0}^{\prime} \xi^{0}\right)^{2}}{c^{2}}}}
$$

In Eq(12), if we multiply $\frac{d \xi^{0}}{\left(1+\frac{a_{0} \xi^{1}}{c^{2}}\right)}$,

$\frac{d \xi^{1}}{1+\frac{a_{0} \xi^{1}}{c^{2}}}=\frac{a_{0}^{\prime} \xi^{0} d \xi^{0}}{\sqrt{1+\frac{\left(a_{0}^{\prime} \xi^{0}\right)^{2}}{c^{2}}}}$

If we integrate $\mathrm{Eq}(13)$,

$$
\frac{c^{2}}{a_{0}} \ln \left|1+\frac{a_{0} \xi^{1}}{c^{2}}\right|=\frac{c^{2}}{a_{0}},\left(\sqrt{1+\frac{\left(a_{0}^{\prime} \xi^{0}\right)^{2}}{c^{2}}}-1\right)
$$

Hence, if we compute about the coordinate $\xi^{1}$, we can represent the super accelerated motion by

Rindler coordinates $\xi^{1}, \xi^{0}$.

$$
\xi^{1}=\frac{c^{2}}{a_{0}}\left[\exp \frac{a_{0}}{a_{0}}\left(\sqrt{1+\frac{\left(a_{0}^{\prime} \xi^{0}\right)^{2}}{c^{2}}}-1\right)-1\right]
$$

If we insert $\mathrm{Eq}(7)$ in $\mathrm{Eq}(15)$, 


$$
\xi^{1}=\frac{c^{2}}{a_{0}}\left[\exp \frac{a_{0}}{a_{0}},\left(\sqrt{1+\frac{\left(a_{0}^{\prime} \xi^{0}\right)^{2}}{c^{2}}}-1\right)-1\right]=\frac{c t}{\sinh \left(\frac{a_{0} \xi^{0}}{c}\right)}-\frac{c^{2}}{a_{0}}
$$

Hence, we can represent the super accelerated motion about coordinates $c t, \xi^{0}$.

$$
c t=\frac{c^{2}}{a_{0}} \exp \frac{a_{0}}{a_{0}}\left(\sqrt{1+\frac{\left(a_{0}^{\prime} \xi^{0}\right)^{2}}{c^{2}}}-1\right) \sinh \left(\frac{a_{0} \xi^{0}}{c}\right)
$$

If we insert $\mathrm{Eq}(17)$ in $\mathrm{Eq}(5)$, we can represent the super accelerated motion about coordinates $x, \xi^{0}$.

$$
x=\operatorname{ctcoth}\left(\frac{a_{0} \xi^{0}}{c}\right)-\frac{c^{2}}{a_{0}}=\frac{c^{2}}{a_{0}} \exp \frac{a_{0}}{a_{0}},\left(\sqrt{1+\frac{\left(a_{0}^{\prime} \xi^{0}\right)^{2}}{c^{2}}}-1\right) \cosh \left(\frac{a_{0} \xi^{0}}{c}\right)-\frac{c^{2}}{a_{0}}
$$

\section{CONCLuSion}

In the general relativity theory, we discover formulas that the super accelerated matter moves with the acceleration in an uniformly accelerated frame.

\section{REFERENCE}

[1] S.Weinberg, Gravitation and Cosmology (John wiley \& Sons,Inc, 1972)

[2] P.Bergman, Introduction to the Theory of Relativity (Dover Pub. Co.,Inc., New York,1976), Chapter V

[3] C.Misner, K,Thorne and J. Wheeler, Gravitation(W.H.Freedman \& Co.,1973)

[4] S.Hawking and G. Ellis,The Large Scale Structure of Space-Time(Cam-bridge University Press,1973)

[5] R.Adler, M.Bazin and M.Schiffer, Introduction to General Relativity(McGraw-Hill,Inc.,1965)

[6] M.Schwarzschild, Structure and Evolution of the Stars (Princeton University Press, 1958; reprint, Dover, N.Y.1965), chapter II

[7] S.Chandrasekhar, Mon,Not.Roy.Astron.Soc.95.207(1935)

[8] C.Rhoades," Investigations in the Physics of Neutron Stars", doctoral dissertation, Princeton University

[9] J.Oppenheimer and H.Snyder, phys.Rev.56,455(1939)

Citation: Sangwha-Yi, (2019). Super Accelerated Motion in Rindler Spacetime. International Journal of Advanced Research in Physical Science (IJARPS) 6(4), pp.20-22, 2019.

Copyright: (๑) 2019 Authors, this is an open-access article distributed under the terms of the Creative Commons Attribution License, which permits unrestricted use, distribution, and reproduction in any medium, provided the original author and source are credited. 